\title{
LV. Extract of a memoir upon hair. Read at the french national institute, by M. Vauquelin
}

\section{Vauquelin}

To cite this article: M. Vauquelin (1806) LV. Extract of a memoir upon hair. Read at the french national institute, by M. Vauquelin, Philosophical Magazine Series 1, 25:100, 351-356, DOI: 10.1080/14786440608563461

To link to this article: http://dx.doi.org/10.1080/14786440608563461

曲 Published online: 18 May 2009.

Submit your article to this journal $[\pi$

Џ Article views: 2

Q View related articles $\square$ 


\section{[ 351$]$}

LV. Extract of a Memoir upon Hair. Read at the French National Institute, by M. VAuquelin *

The principal object which the author had in'view on undertaking his experiments on the above subject, was to ascertain the nature of the animal matter of which hairs are formed, and if there was any thing analogous in the animal œeconomy. But in the course of his experiments phænomena presented themselves which, appearing foreign to the principal substance, led him further than he intended: it did not enter into his plan at first to inquire into the cause of the various colours of hair, which nevertheless became the principal object with which he was occupied. It is only, he says, after labouring a long time upon the same object, by carefully observing the phænomena which arise, and by meditating on the causes which produced them, that we arrive at results often impossible to foresee $\grave{a}$ priori. Nevertheless, he does not flatter himself that he has penetrated into all the secrets of nature on this subject; nor does he propose his ideas but with that reserve which ought to be shown in such difficult researches. He gives, however, an exact description of his experiments ; compares and discusses them, and draws such conclusions as appear to him the most natural. We. shall now give an abridgment of the chief of his experiments, as well as the corollaries he deduces from them.

I boiled, says he, some hair in water for several days, without being able to dissolve it ; the water, however, contained a small quantity of animal matter, as was demonstrated on the application of infusion of galls and other reagents.

It is probable that this matter, which gives the water the property of putrefying, is foreign to the proper substance of hairs. I conclude from this experiment, that at the temperature at which water boils in the air, hairs cannot be dissolved.

* From Annales de Chimie, tome iviii. p. 41. Messrs. Chevruil and Caballe, two of M. Vauquelin's pupils, assisted at the experiments related in the above niemoir. 
I succeeded in dissolving them without altcration in Papin's digester, by regulating the heat properly. If in this operation a certain degree of temperature is exceeder, the substance of the hair is decomposed in whote or in part; circumstance demonstrated by the ammonia, carbonic acid, and the fetid empyreumatic oil found in the solution, to which the oil communicates a deep yellow colour.

In the one or the other case, sulphureted hydrogen gas is liberated in great quantity, which acted strongly upon the copper of the digester, which it blackened: more was found when the heat was raised; which seems to indicate that this substance is produced during the operation.

If we operate upon black hair, or if the heat is not suffcient to decompose it, there remains a black matter, which, on account of its minute division and the consistency of the solution, is deposited very slowly. This matter is principally composed of a black oil, as thick as bitumen, little soluble in alcohol and the alkalis of iron and sulphur perhaps united with each other. Red hair leaves a yellowish red residue, containing plenty of oil, sulphur, and a little iron.

The solutions are almost entirely colourless when they are filtered; the concentrated acids make them turbid, the weak acids produce no change : an excess of these agents restores the liquor its former transparency. Tincture of galls and the oxygenated muriatic acid form abundant precipitates. Silver is blackened in it; the acetate of lead is precipitated from it brown. These solutions, evaporated with every necessary precaution, were not jellied, and only furnished a viscous and gluey matter; whence I concluded that the substance of hair is not gelatinous.

The acids form precipitates more abundant and higher coloured in a solution of hair made at a higher temperature, for this reason, that they decompose an ammoniacal soap, which does not take place in the former case.

I likewise dissolved black and red hair in water containing only four per cent. of caustic potash. During this solution hydrosulphuret of ammonia is liberated : which seems to announce a commencement of decomposition in black 
hair, leaving a black residte formed of thick oil, a little more animalized, and of iron and sulphur. There remains, after the solution of red hair, a yellow oil, containing sulphur and an atom of iron.

The acids form in these solutions white precipitates, soluble in an excess of the above menstrua. These precipitates being redissolved in the acids, there appeared upon the liquor, after some time, an oil under the form of a prismaticcoloured pellicle.

The solution of hair in potash precipitates lead of a black colour, owing to the hydrosulphuret it contains ; that of red hair appears to contain more of it. When they are freed from the sulphur by exposure to the air, they have merely the smell of soap, and become frothy in the same manner.

The acids act upon hair each in their own manner : the sulphuric acid and the muriatic acid assume at first a very fine rose colour, and afterwards dissolve it. The nitric acid makes it yellow, and dissolves it also by means of a gentle heat : the solution presents at its surface a black oil when black hair is used, and a red oil when red hair is made use of. Both the one and the other of these oils become white after some time, and become concrete by cold.

This same solution evaporated properly yields a good deal of oxalic acid, and the uncrystallizable mother-water contains a bitter substance, plenty of iron, and sulphuric acid coming from the sulphur of the hair.

The solution of red hair in the nitric acid contains less iron, but more sulphuric acid than that of black hair.

The oxygenated muriatic acid gas whitens hair at first, afterwards softens it, and reduces it to the form of a viscous and transparent paste like turpentine. This matter is bitter; it is partly dissolved in water and partly in alcohol.

I obtained from hair submitted to the fire in a close apparatus the same products as from every other animal matter, with this difference; that it furnished more sulphur and yielded but very little gas : it left in the retort from 28 to 30 per cent. of charcoal.

By ineineration hair furnished iron and manganese, which Vol, 25. No, 100. Sept.180.6 A a gave 
gave a brownish yellow colour to the ashes, phosphate sulphate, and carbonate of lime, a little muriate of soda, and a considerable quantity of silex. The ashes of red hair are less coloured, because they contain less iron and manganese; those of white hair contain less also, but abundance of magnesia is found in them relatively to the other principles. Hair leaves little more than 1.5 of ashes.

Alcohol extracts two kinds of oil from black hair; the one white, which is deposited upon cooling in the form of small brilliant flakes; the other, which separates in proportion as the alcohol volatilizes, is of a greenish gray, and also becomes concrete in time.

Red hair also yields a white and concrete oil like spermaceti ; but alcohol leaves a deposit by evaporation of another oil, which is as red as blood. What is remarkable and interesting in this experiment is, that the reddest hairs upon which alcohol was employed became brown or deep chestnut-coloured. I conclude from this, that the colour of red hair is owing to the presence of this oil.

According to the experiments reported in this memoir of M. Vauquelin, (a great many of which we have omitted, as being only accessary to the principal object,) it appears that black hair is formed of nine different substances, viz.

1. An animal matter, forming the greatest proportion.

2. A white concrete oil, in small quantity.

3. Another greenish gray oil, very abundant.

4. Iron, the state of which in hair is as yet uncertain.

5. Some particles of oxide of manganese.

6. Phosphate of lime.

7. Carbonate of lime, in a very small quantity.

8. Silex, in a notable quantity.

9. Lastly, a considerable quantity of sulphur.

The same experiments ascertained that red hair does not differ from black, except that it contains a red oil in place of a greenish-black one; finally, that white hair differs from the two former, in so far as its oil is almost colonrless, and it contains phosphate of magnesia, which is not found in the others.

According to this knowledge of the nature of the consti- 
tuent principles of hair, M. Vauquelin thinks that the various colours which distinguish this substance may be accounted for. The black colour, according to him, is produced by a black and bituminous-like oil, and perhaps also by the combination of sulphur with iron. The red and flaxen colours are owing to the presence of a red or yellow oil; the intensity of which diminished by a small quantity of brown oil makes the hair red. Lastly, the white is owing to the absence of the black oil and sulphuretted iron. He thinks, that in red and yellow hair, as well as in white, there is always an excess of sulphur; since when the white metallic oxides are applied to it, such as those of mercury, lead, bismuth, \&ce, they become black very speedily. The manner in which this body acts upon metallic substances makes him think that it is united to hydrogen.

M. Vauquelin endeavours afterwards to explain the whiteness which suddenly takes place in the hair of such persons as are struck with profound grief, or surprised by great fear. It must be supposed, he says, in order to explain this phænomenon, that at the moment when nature is in a state of revolution, and when consequently the natural functions are suspended or changed in their nature, an agent is developed in the animal œconomy, which, passing into the hair, decomposes the colouring matter of it.

But what agent can produce this effect? The acids appear to him to be alone capable of it : this at least is certain, that black hair plunged some time in these menstrua, and particularly in the oxygenated muriatic acid, becomes white very sensibly.

The rapid production of an acid in the animal œconomy does not appear impossible to him, upon considering that a movement of passion in men, as well as in animals, is sufficient to change the nature of certain of their humours and render them poisonous; and secing that the Galvanic fluid often determines in animal and vegetable matters the formation of an acid or an alkali, according to circumstances. As to the whiteness which hair gradually undergoes from age, he attributes it to the want of secretion of the colouring malter.

Independently of the animal matter which forms the A a 9

basis, 
basis, there is in hair a colouring matter which may be separated from it, and the shade of it varies according to the colour it shows. It is to this fat substance that M. Vauquelin attributes the pliability, the elasticity, and the inalterability of hair. It is certainly also to the same substance that they owe the property of burning so rapidly, and forming soap so abundantly with the alkalis.

After having treated the colouring matter of hair, he endeavours to characterize the animal substance which forms it, by comparing it with all those already known. Without detailing all the experiments he made with this view, we shall only say that it was not gelatine; since the solution in water, which is done with much trouble, never becomes a jelly after evaporation: neither is it albumen; because it cannot be dissolved in boiling water without being decomposed, and the solutions act differently with the reagents.

The humour which the substance of hair approaches most, if not absolutely the same, is, according to M. Vauquelin, that which physiologists denominate mucus, or animal mucilage, which is neither gelatine nor albumen.

This humour, which is separated in the nostrils, the mouth, the csophagus, the tracheal artery, the stomach, the bladder, and in general in all the cavities of the body, gives a great deal of viscosity to water, and also the property of frothing very strongly by agitation. In certain coriza it becomes thready, like the substance of silk or spiders' webs; preserves its transparency and flexibility after desiccation; and M. Vauqualin does not doubt that if it contained a little oil, it would completely resemble the substance of hair.

The epidermis, the nails, corns, wool, and down in general are formed of the same animal mucus, and cqually contain in their composition a certain quantity of oil, which sives them their elasticity and pliability.

The beginning of some experiments undertaken by M. Vauquelin upon the humour of the plica polonica, which was furnished him by M. Alibert, physician of the hospital of St. Louis, indnced him to think that it was. of the same nature as the substance of hair ; and that it is what is superabundant to the formation of the latter. 